\title{
Minimal Flavour Mixing of Quarks and Leptons
}

\author{
J.L. Chkareuli \\ Institute of Physics, Georgian Academy of Sciences, 380077 Tbilisi, Georgia
}

\begin{abstract}
Present situation in the flavour mixing of quarks and leptons is briefly reviewed and a new approach called the Minimal Flavour Mixing (MFM) is considered in detail. According to MFM the whole of the flavour mixing is basically determined by the physical mass generation of the first family of fermions. So, in the chiral symmetry limit when the masses of the lightest quarks, $u$ and $d$, vanish, all the weak mixing angle vanish. This minimal pattern is shown to fit extremely well the already established CKM matrix elements and to give fairly distinctive predictions for the as yet poorly known ones. Remarkably, together with generically small quark mixing it also leads to the large neutrino mixing thus giving adequate solution to the solar and atmospheric neutrino oscillation problem. The possible origin of this approach in the MSSM extended by the high-scale $S U(3)_{F}$ chiral family symmetry is discussed.
\end{abstract}

Plenary talk given at the SUSY'01: 9th International Conference on Supersymmetry and Unification of Fundamental Interactions (11-17 June 2001, JINR, Dubna, Russia) 


\section{Preamble}

The flavour mixing of quarks and leptons is certainly one of the major problems that presently confront particle physics. Many attempts have been made to interpret the pattern of this mixing in terms of various family symmetries - discrete or continuous, global or local (for a recent review see [[]]). However, despite some progress in understanding the flavour mixing problem, one has the uneasy feeling that, in many cases, the problem seems just to be transferred from one place to another. The peculiar quark-lepton mass hierarchy is replaced by a peculiar set of flavour charges assigned to fermions or a peculiar hierarchy of the extra Higgs field VEVs. As a result, apart from an original Cabibbo mixing there are not in fact distinctive and testable generic predictions concerning the flavour mixing angles, especially those for the heaviest quarks $t$ and $b$. Meanwhile, the present observational status of quark flavour mixing shows[2] that the third family $t$ and $b$ quarks are largely decoupled from the lighter families. At first sight, it looks quite surprising that not only the 1-3 "far neighbour" mixing $\left(\theta_{13}\right)$ but also the 2-3 "nearest neighbour" mixing $\left(\theta_{23}\right)$ happen to be small compared with an "ordinary" 1-2 Cabibbo mixing $\left(\theta_{12}\right)$ which is determined, according to common belief, by the lightest $u$ and $d$ quarks. This would mean that all the other mixings could also be controlled by the lightest masses $m_{u}$ and $m_{d}$. So, in the chiral symmetry limit $m_{u}=m_{d}=0$ all the flavour mixings disappear.

Recently, in accordance with that view a new mechanism of flavour mixing, which we called Minimal Flavour Mixing (MFM), was proposed [3] (see the papers in [4] for further discussion). Remarkably, together with generically small quark mixing this picture leads to the large neutrino mixing provided that neutrino masses appear by themselves through a known "see-saw" mechanism and the neutrino Dirac and Majorana masses have the hierarchical structure like as masses of ordinary quarks and leptons.

\section{Where does flavour mixing come from?}

\subsection{Gauge (unrotated) and physical quarks}

Without much loss in generality we could choose the starting mass matrices of quarks as those being Hermitian and having one texture zero. 


\subsubsection{Upper quarks}

$$
\begin{aligned}
u^{0}, c^{0}, t^{0} & \Longleftrightarrow u, c, t \\
\left(\begin{array}{lll}
0 & X & Y \\
X^{*} & m_{c}^{0} & Z \\
Y^{*} & Z^{*} & m_{t}^{0}
\end{array}\right) & \Leftrightarrow\left(\begin{array}{lll}
m_{u} & 0 & 0 \\
0 & m_{c} & 0 \\
0 & 0 & m_{t}
\end{array}\right)
\end{aligned}
$$

2.1.2 Down quarks

$$
\begin{aligned}
d^{0}, s^{0}, b^{0} & \Longleftrightarrow d, s, b \\
\left(\begin{array}{lll}
0 & X^{\prime} & Y^{\prime} \\
X^{\prime *} & m_{s}^{0} & Z^{\prime} \\
Y^{\prime *} & Z^{\prime *} & m_{b}^{0}
\end{array}\right) & \Longleftrightarrow\left(\begin{array}{lll}
m_{d} & 0 & 0 \\
0 & m_{s} & 0 \\
0 & 0 & m_{b}
\end{array}\right)
\end{aligned}
$$

2.1.3 The measure of Flavour Mixing (in terms of mass deviations)

Proposing that the difference between masses of the physical and gauge quarks of a given generation is much less than the mass of quark of a previous generation

$$
\begin{aligned}
& m_{t}-m_{t}^{0} \quad \ll m_{c}, \quad m_{c}-m_{c}^{0} \ll m_{u} \quad \Longrightarrow \quad m_{t}-m_{t}^{0} \simeq m_{u} \\
& m_{b}-m_{b}^{0} \quad \ll \quad m_{s}, \quad m_{s}-m_{s}^{0} \ll m_{d} \quad \Longrightarrow \quad m_{b}-m_{b}^{0} \simeq m_{d}
\end{aligned}
$$

we come to the MFM :

Flavour Mixing is basically determined by the lightest family mass generation.

\section{The prototype quark mixing: 2 alterna- tives}

The proposed MFM driven solely by the generation of the lightest family mass could actually be realized in two generic ways. 


\subsection{Scenario A: " $u$ and $d$ quark masses running along the diagonal"}

The first way is when the lightest family mass $\left(m_{u}, m_{d}\right.$ or $\left.m_{e}\right)$ appears as a result of the complex flavour mixing of all three families. It "runs along the main diagonal" of the corresponding $3 \times 3$ mass matrix $M$, from the basic dominant element $M_{33}$ to the element $M_{22}$ (via a rotation in the 2-3 sub-block of $M$ ) and then to the primordially texture zero element $M_{11}$ (via a rotation in the 1-2 sub-block). The direct flavour mixing of the first and third families of quarks and leptons is supposed to be absent or negligibly small in $M$. Let us note that the "running along the diagonal" of the lightest mass means mathematically the proportionality condition between diagonal and off-diagonal matrix elements in the $M$ of the type:

$$
M_{22}: M_{33}=\left|M_{12}\right|^{2}:\left|M_{23}\right|^{2}
$$

\subsubsection{Upper quarks}

Doing so we inescapably come to the mass-matrix for the upper quarks of the type (using that the trace and determinant of the Hermitian matrix gives the sum and product of its eigenvalues)

$$
M_{13}^{U}=M_{31}^{U}=0 \Longrightarrow\left(\begin{array}{lll}
0 & \sqrt{m_{u} m_{c}} e^{i \alpha} & 0 \\
\sqrt{m_{d} m_{s}} e^{-i \alpha} & m_{c} & \sqrt{m_{u} m_{t}} e^{i \beta} \\
0 & \sqrt{m_{u} m_{t}} e^{-i \beta} & m_{t}-m_{u}
\end{array}\right)
$$

\subsubsection{Down quarks}

Analogously, the mass matrix for the down quarks looks like

$$
M_{13}^{D}=M_{31}^{D}=0 \Longrightarrow\left(\begin{array}{lll}
0 & \sqrt{m_{d} m_{s}} e^{i \alpha^{\prime}} & 0 \\
\sqrt{m_{d} m_{s}} e^{-i \alpha^{\prime}} & m_{s} & \sqrt{m_{d} m_{b}} e^{i \beta^{\prime}} \\
0 & \sqrt{m_{d} m_{b}} e^{-i \beta^{\prime}} & m_{b}-m_{d}
\end{array}\right)
$$

\subsubsection{Weak mixing angles}

So, now all the weak mixing angles can be calculated in terms of the quark mass ratios and $\mathrm{CP}$-violating phase (which tends to be maximal to give the right value of the Cabibbo angle) 


$$
\begin{array}{ll}
\left|s_{12}\right| \simeq\left|\sqrt{\frac{m_{d}}{m_{s}}}-e^{i\left(\alpha-\alpha^{\prime}\right)} \sqrt{\frac{m_{u}}{m_{c}}}\right| \quad\left(\text { CP phase } \delta=\alpha-\alpha^{\prime} \text { tends to } \pi / 2\right) \\
\left|s_{23}\right|=\left|\sqrt{\frac{m_{d}}{m_{b}}}-e^{i\left(\beta-\beta^{\prime}\right) \sqrt{\frac{m_{u}}{m_{t}}}}\right| \quad\left(\begin{array}{c}
0.038(7) / 0.039(3) \\
t h / \exp
\end{array}\right) \\
\left|\frac{s_{12}}{s_{23}}\right|=\sqrt{\frac{m_{u}}{m_{c}}}=0.05(1)
\end{array}
$$

\subsection{Scenario B: " $m_{u}$ walking around the corner, while $m_{d}$ runs along the diagonal"}

The second way, on the contrary, presupposes direct flavour mixing of just the first and third families. There is no involvement of the second family in the mixing. In this case, the lightest mass appears in the primordially texture zero $M_{11}$ element "walking round the corner" (via a rotation in the 1-3 sub-block of the mass matrix $M$ ). Certainly, this second version of the MFM mechanism cannot be used for both the up and the down quark families simultaneously, since mixing with the second family members is a basic part of the CKM phenomenology (Cabibbo mixing, non-zero $V_{c b}$ element, CP violation). However, this second way could work for the up quark family provided that the down quarks follow the first way.

\subsubsection{Upper quarks}

Now we have the new matrix for the upper quarks

$$
M_{12}^{U}=M_{21}^{U}=M_{23}^{U}=M_{32}^{U}=0 \Longrightarrow\left(\begin{array}{lll}
0 & 0 & \sqrt{m_{u} m_{t}} e^{i \alpha} \\
0 & m_{c} & 0 \\
\sqrt{m_{u} m_{t}} e^{-i \alpha} & 0 & m_{t}-m_{u}
\end{array}\right)
$$

\subsubsection{Down quarks}

While for the down quarks the above matrix (6) is remained

$$
M_{13}^{D}=M_{31}^{D}=0 \Longrightarrow\left(\begin{array}{lll}
0 & \sqrt{m_{d} m_{s}} e^{i \alpha^{\prime}} & 0 \\
\sqrt{m_{d} m_{s}} e^{-i \alpha^{\prime}} & m_{s} & \sqrt{m_{d} m_{b}} e^{i \beta^{\prime}} \\
0 & \sqrt{m_{d} m_{b}} e^{-i \beta^{\prime}} & m_{b}-m_{d}
\end{array}\right)
$$


so that the weak mixing angles and CP-violation phase are given by new formulas.

\subsubsection{Weak mixing angles}

Actually, the mass matrices (8) and (9) lead to the simplest expressions which ever have been derived for CKM angles

$$
s_{12} \simeq \sqrt{\frac{m_{d}}{m_{s}}}, \quad s_{23} \simeq \sqrt{\frac{m_{d}}{m_{b}}} \quad, \quad s_{13} \simeq \sqrt{\frac{m_{u}}{m_{t}}}
$$

while the $\mathrm{CP}$ violation phase $\delta=\alpha_{U}-\alpha^{\prime}+\beta^{\prime}$ is left yet arbitrary.

\subsection{CKM matrix}

Our numerical results for both versions of our model, with a maximal CP violating phase (see discussion in Section 5), are summarized in the following CKM matrix:

$$
V_{C K M}=\left(\begin{array}{ccc}
0.975(1) & 0.222(4) & 0.0023(5) A \\
& & 0.0036(6) B \\
0.222(4) & 0.975(1) & 0.038(4) \\
0.009(2) & 0.038(4) & 0.999(1)
\end{array}\right)
$$

The uncertainties in brackets are largely given by the uncertainties in the quark masses (calculated at the electroweak scale[0]). There is clearly a real and testable difference between scenarios A and B given by the value of the $V_{u b}$ element. Everyone can see when looking into Particle Physics Booklet that the MFM ansatz perfectly works. The distinctive predictions for the presently relatively poorly known $V_{u b}$ and $V_{t d}$ elements should be tested

in the nearest future. Meanwhile, the present data from CLEO [6] seem to favour scenario B.

\section{Lepton sector}

The lepton mixing matrix is defined analogously to the CKM matrix:

$$
U=U_{\nu} U_{E}^{\dagger}
$$


where the indices $\nu$ and $E$ stand for $\nu=\left(\nu_{e}, \nu_{\mu}, \nu_{\tau}\right)$ and $E=(e, \mu, \tau)$. Our model predicts the small charged lepton mixing angles in the matrix $U_{E}$. They do not markedly effect atmospheric neutrino oscillations [7], which appear to require essentially maximal mixing $\sin ^{2} 2 \theta_{\text {atm }} \simeq 1$. It follows then that the large neutrino mixing responsible for atmospheric neutrino oscillations should mainly come from the $U_{\nu}$ matrix associated with the neutrino mass matrix in (12). This requires a different mass matrix texture for the neutrinos compared to the charged fermions. Remarkably, there appears to be no need in our case for some different mechanism to generate the observed mixing pattern of neutrinos: they can get physical masses and mixings via the usual "see-saw" mechanism [8]

$$
M_{\nu}=-M_{N}^{T} M_{N N}^{-1} M_{N}
$$

using the proposed MFM mechanism for their primary Dirac and Majorana masses, $M_{N}$ and $M_{N N}$, respectively. So, we also have two possible scenarios in the lepton sector as well.

\subsection{Scenario A*: "all the lightest lepton Dirac and Majorana masses running along the diagonal"}

\subsubsection{Charged leptons}

For the charged leptons we have according to the MFM the mixing angles:

$$
\sin \theta_{e \mu}=\sqrt{\frac{m_{e}}{m_{\mu}}} \quad \sin \theta_{\mu \tau}=\sqrt{\frac{m_{e}}{m_{\tau}}} \quad \sin \theta_{e \tau} \simeq 0 \quad\left(\text { in } U_{C K M}^{l e p}=U_{\nu} U_{E}^{\dagger}\right)
$$

It follows then that the large neutrino mixing responsible for atmospheric neutrino oscillations should mainly come from the neutrino mass matrix by itself.

\subsubsection{Neutrinos}

Let us parametrize the neutrino Dirac mass matrix $M_{N}$ as follows

$$
M_{N} \simeq M_{N 3}\left(\begin{array}{lll}
0 & y^{3} & 0 \\
y^{3} & y^{2} & y^{2} \\
0 & y^{2} & 1
\end{array}\right)
$$


proposing the hierarchy like as that for down quarks $\left(y \approx\left(m_{s} / m_{b}\right)^{1 / 2} \approx\right.$ 0.15 ), while for the Majorana mass matrix $M_{N N}$ (for right-handed neutrinos) we take the stronger hierarchy of the type 9

$$
M_{N N} \simeq M_{N N 3}\left(\begin{array}{lll}
0 & y^{5} & 0 \\
y^{5} & y^{4} & y^{3} \\
0 & y^{3} & 1
\end{array}\right)
$$

which is similar to that for the upper quarks. However, the basic proportionality condition (4) underlying the MFM mechanism is satisfied for both. Now, constructing the mass matrix $M_{\nu}$ (13) for physical neutrinos one immediately comes to the characteristic predictions for the standard two-flavour atmospheric and solar neutrino oscillation parameters 9

$$
\sin ^{2} 2 \theta_{\text {atm }} \simeq 1, \sin ^{2} 2 \theta_{\text {sun }} \simeq \frac{2}{3}, U_{e 3} \simeq \frac{1}{2 \sqrt{2}} y, \frac{\Delta m_{\text {sun }}^{2}}{\Delta m_{\text {atm }}^{2}} \simeq \frac{1}{4} y^{2}
$$

as compared with the experimentally allowed intervals (for the large-angle MSW oscillation solution to the solar neutrino problem)

$$
\begin{aligned}
0.82 & \leq \sin ^{2} 2 \theta_{\text {atm }} \leq 1,0.65 \leq \sin ^{2} 2 \theta_{\text {sun }} \leq 1 \\
\left|U_{e 3}\right|^{2} & \leq 0.05,5 \cdot 10^{-3} \leq \frac{\Delta m_{\text {sun }}^{2}}{\Delta m_{\text {atm }}^{2}} \leq 5 \cdot 10^{-2}
\end{aligned}
$$

\subsection{Alternative B": "the lightest Majorana mass walk- ing around the corner, while the lightest Dirac masses run along the diagonal"}

Again, we take for the neutrino Dirac mass matrix $M_{N}$ the form (15), while for the Majorana mass matrix $M_{N N}$ we take the alternative II form

$$
M_{N N} \simeq M_{N N 3}\left(\begin{array}{lll}
0 & 0 & y^{q} \\
0 & y^{p} & 0 \\
y^{q} & 0 & 1
\end{array}\right)
$$

with an arbitrary eigenvalue hierarchy of the type

$$
M_{N N 3}: M_{N N 2}: M_{N N 1} \simeq 1: y^{p}: y^{2 q}
$$


The seesaw formula (13) then generates an effective physical neutrino mass matrix $M_{\nu}$ which automatically leads to the large (maximal) $\nu_{\mu}-\nu_{\tau}$ mixing and small $\nu_{e}-\nu_{\mu}$ mixing for any hierarchy in the Majorana mass matrix (19) satisfying the condition $p \geq 2 q-1$ mentioned above. Taking, for an example, $p=5$ and $q=3$ one naturally comes to the following predictions for the two flavour oscillation parameters

$$
\sin ^{2} 2 \theta_{\text {atm }} \simeq 1, \quad \sin ^{2} 2 \theta_{\text {sun }} \simeq \frac{2}{9} y^{2}, \quad U_{e 3} \simeq \frac{1}{\sqrt{2}} y, \quad \frac{\Delta m_{\text {sun }}^{2}}{\Delta m_{\text {atm }}^{2}} \simeq \frac{9}{16} y^{2}
$$

For the known value of the hierarchy parameter $y$ (see above) our predictions (21) turn out to be inside of the experimentally allowed intervals [10] for the small-angle MSW solution for the solar neutrino oscillation:

$$
\begin{aligned}
0.82 & \leq \sin ^{2} 2 \theta_{\text {atm }} \leq 1, \quad 10^{-3} \leq \sin ^{2} 2 \theta_{\text {sun }} \leq 10^{-2} \\
\left|U_{e 3}\right|^{2} & \leq 0.05, \quad 5 \cdot 10^{-4} \leq \frac{\Delta m_{\text {sun }}^{2}}{\Delta m_{\text {atm }}^{2}} \leq 9 \cdot 10^{-3}
\end{aligned}
$$

Note that in contrast to the LMA case (17), one must include in the SMA case (21) even the small contribution stemming from the charged lepton sector (see Eq. (14) into the solar neutrino oscillations.

So, one can see that the proposed MFM mechanism works quite successfully in the lepton sector as well as in the quark sector. Remarkably, the same mechanism results simultaneously in small quark mixing and large lepton mixing.

\section{Conclusion and outlook}

By its nature, the MFM mechanism is not dependent on the number of quarklepton families nor on any "vertical" symmetry structure, unifying quarks and leptons inside a family as in Grand Unified Theories (GUTs). From the theoretical point of view it is based on the generic proportionality condition (4) between diagonal and off-diagonal elements of the mass matrices. For the $\mathrm{N}$ family case, this condition could be expressed as:

$$
M_{22}: M_{33}: \ldots: M_{N N}=\left|M_{12}\right|^{2}:\left|M_{23}\right|^{2}: \ldots:\left|M_{N-1 N}\right|^{2}
$$


showing clearly that the heavier families (4th, 5th, ...), had they existed, would be more and more decoupled from the lighter ones and from each other. Indeed, this behavior can to some extent actually be seen in the presently observed CKM matrix elements involving the third family quarks $t$ and $b$.

One might think that the condition (23) suggests some underlying flavour symmetry, probably non-abelian $S U(N)$, treating the $\mathrm{N}$ families in a special way. Indeed, for $N=3$ families, we have found[3] that the $S U(3)$ chiral family (or horizontal) symmetry [11], properly interpreted in terms of the symmetry breaking vacuum configurations, leads to the basic condition (14) for the mass matrices of the down quarks and charged leptons leaving the up quark and neutrino Majorana masses to follow scenario B and B*, respectively. One can say that the combined scenario $\mathrm{B}+\mathrm{B}^{*}$ for quark and lepton mixing is certainly favored by the $S U(3)$ symmetry, since in the $S U(3)$ framework the scenario A wants the up and down quark mass matrices to be proportional to each other, while scenario $\mathrm{A}^{*}$ requires the exactly lepton mass-like hierarchy for the Majorana neutrino masses as well, both of which are turned out to be observationally excluded.

At the same time the symmetry-breaking horizontal scalar fields, triplets and sextets of $S U(3)$, develop in general complex VEVs and in cases linked to the MFM mechanism transmit a maximal $\mathrm{CP}$ violating phase $\delta=\frac{\pi}{2}$ to the effective Yukawa couplings. Apart from the direct predictability of $\delta$ (which was used in the numerical analysis of the CKM matrix given in the above), the possibility that CP symmetry is broken spontaneously like other fundamental symmetries of the Standard Model seems very attractive-both aesthetically and because it gives some clue to the flavour part of strong $\mathrm{CP}$ violation. On the other hand, spontaneous $\mathrm{CP}$ violation means that the scale of the $S U(3)$ family symmetry must be rather high (not much less than $\left.M_{G U T}\right)$ in order to avoid the standard domain wall problem by the well-known inflation mechanism.

So, an $S U(3)$ family symmetry seems to be a good candidate for the basic theory underlying our proposed MFM mechanism, although we do not exclude the possibility of other interpretations as well. Certainly, even without a theoretical derivation of Eq.(23), the MFM mechanism can be considered as a successful predictive ansatz in its own right. Its further testing could shed light on the underlying flavour dynamics and the way towards the final theory of flavour. 
We summarize in conclusion the main outputs following from Minimal Flavour Mixing:

* MFM successfully works both in quark and lepton sector;

$\star \star M F M$ can be derived from SU(3) theory of flavour with extra benefits such as the hierarchy in masses and mixings of quarks and leptons and the spontaneous (maximal) CP violation;

$\star \star \star$ From four yet experimentally allowed scenarios $A+A^{*}, A+B^{*}, B+A^{*}$ and $B+B^{*}$ for a combined quark and lepton mixing the scenario $B+B^{*}$ is most preferable as from the observations, so by an accommodation in $S U(3)$ theory of flavour;

$\star \star \star \star$ While the proposed mechanism describes well all the presently available data, the most critical prediction for the $M F M / B+B^{*}$ scenario are left

to be $V_{u b}=\sqrt{\frac{m_{u}}{m_{t}}}$ in the quark mixing and SMA solution to the solar neutrino problem which, if finally confirmed, might allow to enthusiastically say:

"Minimal Flavour Mixing is in fact Standard Flavour Mixing".

\section{Acknowledgements}

Many thanks to organizers of the SUSY'01 for their impressive work and warmest hospitality. I also should like to thank B. Arbuzov, H. Fritzsch, D. Kazakov, A. Kobakhidze, H. Leutwyler, D. Sutherland and Z. -Z. Xing and especially my collaborators C.Froggatt and H. Nielsen for stimulating discussions and useful remarks.

\section{References}

[1] H. Fritzsch and Z. -Z. Xing, Prog. in Part. and Nucl. Phys. 45 (2000) 1.

[2] Particle Data Group, The Europ. Phys. Journ. C 15 (2000) 1.

[3] J.L. Chkareuli and C.D. Froggatt, Phys.Lett. B 450 (1999) 158; 
J.L. Chkareuli, C.D. Froggatt and H.B. Nielsen, Minimal Mixing of Quarks and Leptons in the SU(3) Theory of Flavour, hep-ph/0109156, Nucl. Phys. B (to appear).

[4] K. Matsuda, T. Fukuyama and H. Nishiura, Phys. Rev. D 60 (1999) 013006;

D. Falcone, Mod.Phys.Lett. A 14 (1999) 1989.

T.K. Kuo, S.W. Mansour and G. -H. Wu, Phys. Rev. D 60 (1999) 093004;

Shao-Hsuan Chiu, T.K. Kuo and Guo-Hong Wu, Phys. Rev. D 62 (2000) 053014;

D. Falcone and F. Tramontano, Phys. Rev. D 63 (2001) 073007;

B.R. Desai and A.R. Vaucher, Phys. Rev. D 63 (2001) 113001.

[5] H. Fusaoka and Y. Koide, Phys. Rev. D57 (1998) 3986.

[6] CLEO Coll. (B.H. Behrens et al), Phys. Rev. D61 (2000) 052001.

[7] Super-Kamiokande Coll. (Y. Fukuda et al), Phys. Rev. Lett. 81 (1998) 1562; ibid. 85 (2000) 3999.

[8] M. Gell-Mann, P. Ramond and R. Slansky, in Supergravity, ed. by P. von Nievenhuizen and D.Z. Friedman, N.Holland, 1979.

[9] K. Matsuda, T. Fukuyama and H. Nishiura, see ref. [4].

[10] J.N. Bahcall, P.I. Krastev and A.Yu. Smirnov, Phys. Rev. D 58 (1998) 096016; J.N. Bahcall, M.C. Gonzales-Garcia and C. Pena-Garay, hep$\mathrm{ph} / 0106258$.

[11] J.L. Chkareuli, JETP Lett. 32 (1980) 671;

Z.G. Berezhiani and J.L. Chkareuli, Yad. Fiz. 37 (1983) 1043;

F. Wilczek, preprint NSF-ITP-83-08 (1983);

Z.G. Berezhiani, Phys. Lett. B 129 (1983) 99; ibid B 150 (1985) 177.

J.C. Wu, Phys. Rev. D 36 (1987) 1514.

J.L. Chkareuli, Phys. Lett. B 246 (1990) 498; ibid. B 300 (1993) 361. 